\title{
A New Design Method for PI-PD Control of Unstable Fractional-Order System with Time Delay
}

\author{
Min Zheng $\mathbb{D},{ }^{1,2}$ Tao Huang $\mathbb{D}^{2},{ }^{2}$ and Guangfeng Zhang $\mathbb{D}^{2}$ \\ ${ }^{1}$ School of Mechatronic Engineering and Automation, Shanghai University, \\ Shanghai Key Laboratory of Power Station Automation Technology, 200072 Shanghai, China \\ ${ }^{2}$ School of Mechatronic Engineering and Automation, Shanghai University, 200072 Shanghai, China \\ Correspondence should be addressed to Min Zheng; zhengmin203@163.com
}

Received 25 July 2019; Accepted 4 October 2019; Published 30 October 2019

Guest Editor: Wonhee Kim

Copyright (C) 2019 Min Zheng et al. This is an open access article distributed under the Creative Commons Attribution License, which permits unrestricted use, distribution, and reproduction in any medium, provided the original work is properly cited.

In this paper, a practical PI-PD controller parameter tuning method is proposed, which uses the incenter of the triangle and the Fermat point of the convex polygon to optimize the PI-PD controller. Combined with the stability boundary locus method, the PIPD controller parameters that can ensure stability for the unstable fractional-order system with time delay are obtained. Firstly, the parameters of the inner-loop PD controller are determined by the centre coordinates of the CSR in the $k_{\mathrm{d}}-k_{\mathrm{f}}$ plane. Secondly, a new graphical method is used to calculate the parameters of the PI controller, in which Fermat points in the CSR of $\left(k_{\mathrm{p}}-k_{\mathrm{i}}\right)$ plane are selected. Furthermore, the method is extended to uncertain systems, and the PI-PD controller parameters are obtained by using the proposed method through common stable region of all stable regions. The proposed graphical method not only ensures the stability of the closed-loop system but also avoids the complicated optimization calculations. The superior control performance of this method is illustrated by simulation.

\section{Introduction}

Proportional-integral-derivative (PID) controller has been widely used in industrial control systems for decades because of its simple structure and convenient implementation [1-6]. As the control plant becomes more and more complex, the performance of the controller is required to be higher and higher and the controllability of the system becomes difficult to solve. By using Schade's fixed point theorem, the controllability problem of nonlinear fractional integro-differential dynamical systems is transformed into the fixed point problem in [7]. The integral-order PID (IOPID) controller has limitations in control integration, instability, and delay process, and it often leads to large step response, large overshoot, and large impact, especially for unstable complex systems with time delay, and it is difficult to obtain good closed-loop performance [8-12]. Researchers have proposed a series of controller design schemes, including integerorder PID (IOPID) controller, sliding mode controller (SMC) to fractional-order PID (FOPID) controller, fractional-order sliding mode controller and other complex controllers, and so on [12-19]. The computational cost of these controllers increases geometrically with the change of controller structure complexity, but the performance improvement is not satisfactory. For this reason, researchers put forward a PI-PD controller structure by changing the structure of PID controllers in $[8,9,20]$. The controller converts the unstable open-loop plant into the stable openloop plant through the PD controller of the internal loop circuit, ensuring that the open-loop poles of the resonance and integration processes are in the proper position. The PI controller of the outer loop controls the inner loop of the system as a whole. Many studies have shown that the PI-PD structure has superior closed-loop performance for an unstable system with time delay [20]. The IOPID controller has three adjustable parameters, while the PI-PD controller has four adjustable parameters. Therefore, the PI-PD controller has advantages over the IOPID controller in terms of control structure and control freedom [21, 22]. The traditional controller has a good control effect on the precise model 
with certain parameters. However, in most cases, the exact model of the actual system could not be obtained [23]. Therefore, an excellent controller design must meet the requirements of robustness, stability, control performance, and the ability to overcome the uncertainty of model parameters $[24,25]$.

In practical applications, PID controller parameter tuning methods are divided into three categories: the iterative optimization method based on intelligent optimization $[26,27]$; the setting formula method for specific system $[3,4,28]$; and a parameter selection method [29-34] based on the stable region. The stable boundary locus (SBL) method has been widely studied, which provides an effective solution for some specific systems.

In recent years, PI-PD controllers have been used extensively in systems with unstable processes and time delay. Kaya and Atherton [28] studied the first-order time-delay unstable system of a PI-PD controller by using a simple regulation formula tuning method, taking integral of squared error (ISE) and integral of squared time weighted error (ISTE) as performance indicators. Tan [29] and Srivastava and Pandit [30] proposed a graphic method based on SBL by using gain phase tester to achieve the specified gain and phase margin and extended this method to the systems with parameter uncertainty by Kharitonov theorem. For the systems with uncertain timevarying delays, Shariati proposed a neutral system approach to stabilize and synthesize $H_{\infty}$ PI-PD controllers. A new bounded real lemma was given for neutral systems with efficiency in designing the $H_{\infty}$ controllers with both state delay and state derivative delay coefficients depending on the controller parameters [35]. Onat et al. $[31,32]$ proposed the concept of the weight geometric centre (WGC) method based on stable domain, which was applied to the parameter design of a PI controller. Ozyetkin [36] extended the WGC method to the fractional-order PI-PD. All the sample points that make up the stable locus boundary would be used to calculate the coordinate of WGC point. However, it has the large calculation cost which is not conducive to the application of real-time system control. In addition, under fractionalorder control, WGC points do not always fall into the stable region. Onat [34] simplified the WGC method and constructed a convex stabilization region with only three points to ensure that the WGC is in the stable region.

However, the above literature only considered integerorder systems. On the other hand, there is little research on the PI-PD controller design through these graphical parameter selection methods. Inspired by the above analysis, the main contribution of this paper is to solve the parameters of the general fractional-order time-delay system PI-PD controller by the graphical method and generalize it to the parameter uncertainty system. In this paper, a new method to tune the parameters of PI-PD is proposed by using the incenter point, Fermat point, and geometry method in convex stable region (CSR). The main design process includes the following steps: firstly, for the open-loop uncertain system, the internal loop PD controller is designed. The stability boundary locus on the $k_{\mathrm{d}}-k_{\mathrm{f}}$ plane is obtained by using the internal loop closedloop characteristic polynomial. Then, the incenter coordinates of the CSR are computed. Secondly, embedding PD controller parameters into transfer function, desired stability boundary locus of $\mathrm{Pl}$ controller is computed by the same procedure. Then, a quadrilateral Fermat point in CSR of the outer loop is obtained which is the parameter of the PI controller. This method only needs simple geometric calculation to obtain the parameters of the PIPD controller, which is solved in the stable region to ensure the closed-loop stability. It uses several special points of the stable region and fewer points describing the coordinates than the WGC method, ensuring that the calculated points are situated in the stable region and the PI-PD controller has superior robustness. The simulation example shows that the method presented in this paper has a satisfactory control effect.

The structure of this paper is organized as follows. In Section 2, the problem statements of the controlled plant and controller are given, and then the proposed method is presented and extended to uncertain systems. Simulative and experimental application examples are considered to illustrate the effectiveness of the proposed method in Section 3. Section 4 gives conclusion.

\section{Main Results}

2.1. Problem Statement. The generalized PI-PD negative feedback system shown in Figure 1, where $r$ is the set point and $y$ is the negative feedback output. The generalized fractional transfer function $G_{p}(s)$ is written as follows:

$$
G_{p}(s)=\frac{N_{p}(s)}{D_{p}(s)} e^{-\tau s},
$$

where $\tau$ is the time delay and $N_{p}(s)$ and $D_{p}(s)$ are the numerator and the denominator of the transfer function, respectively. They are defined with the forms:

$$
\begin{aligned}
& N_{p}(s)=b_{0} s^{\beta_{0}}+b_{1} s^{\beta_{1}}+\cdots+b_{n-1} s^{\beta_{n-1}}+b_{n} s^{\beta_{n}}=\sum_{i=0}^{n} b_{i} s^{\beta_{i}}, \\
& D_{p}(s)=a_{0} s^{\alpha_{0}}+a_{1} s^{\alpha_{1}}+\cdots+a_{n-1} s^{\alpha_{n}}+a_{n} s^{\alpha_{n}}=\sum_{i=0}^{n} a_{i} s^{\alpha_{i}},
\end{aligned}
$$

where $a_{j}, b_{i}, \alpha_{j}, \beta_{i}, i, j=0,1, \ldots, n$ are real numbers and $\alpha_{n}>\alpha_{n-1}>\cdots>\alpha_{2}>\alpha_{1}>\alpha_{0} \geq 0, \beta_{n}>\beta_{n-1}>\cdots>\beta_{2}>\beta_{1}>$ $\beta_{0} \geq 0, \alpha_{n}>\beta_{n}$. The internal loop PD controller $C_{\mathrm{PD}}(s)$ and external loop PI controller $C_{\mathrm{PI}}(s)$ are defined as

$$
\begin{aligned}
C_{\mathrm{PD}}(s) & =\frac{N_{\mathrm{PD}}(s)}{D_{\mathrm{PD}}(s)}=k_{\mathrm{f}}+\frac{k_{\mathrm{d}} s}{T s+1}, \\
C_{\mathrm{PI}}(s) & =\frac{N_{\mathrm{PI}}(s)}{D_{\mathrm{PI}}(s)}=k_{\mathrm{p}}+\frac{k_{\mathrm{i}}}{s},
\end{aligned}
$$

where $T=0.01, k_{\mathrm{f}}$ and $k_{\mathrm{d}}$ are proportional and differential gains of the internal loop PD controller, and $k_{\mathrm{p}}$ and $k_{\mathrm{i}}$ are proportional and integral gains of the outer-loop PI controller, respectively. 


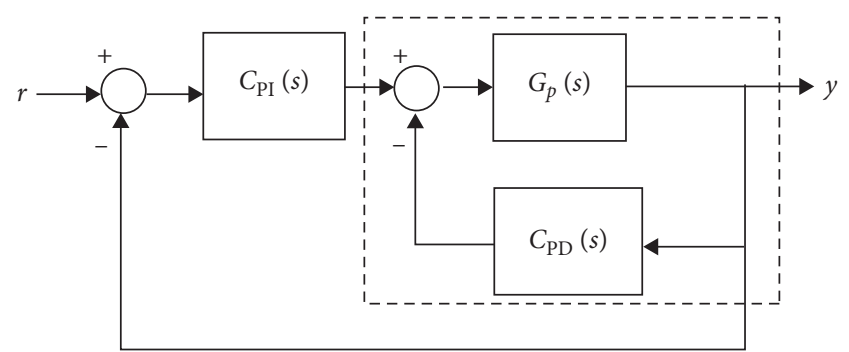

Figure 1: The PI-PD control system for generalized fractional plant.

The main purpose is to solve the parameters of the generalized fractional-order time-delay system (1) with PIPD controller (3) and (4) by the graphical method.

2.2. The Proposed Method. The PI-PD controller has four adjustable parameters $\left(k_{\mathrm{f}}, k_{\mathrm{d}}, k_{\mathrm{p}}, k_{\mathrm{i}}\right)$ that need to be determined. The internal PD controller is used to change the poles of the plant transfer function $G_{p}(s)$ to more desirable locations for control by the outer-loop PI controller.

Substituting $s^{\alpha}=\omega^{\alpha}(\cos (\alpha \pi / 2)+j \sin (\alpha \pi / 2))$ into (1) and dividing the numerator and denominator into a real part and an imaginary part, one can write

$$
\frac{N_{p}(j \omega)}{D_{p}(j \omega)}=\frac{N_{\mathrm{PE}}(j \omega)+j N_{\mathrm{PO}}(j \omega)}{D_{\mathrm{PE}}(j \omega)+j D_{\mathrm{PO}}(j \omega)} .
$$

Simplify $N_{\mathrm{PE}}(j \omega), N_{\mathrm{PO}}(j \omega), D_{\mathrm{PE}}(j \omega)$, and $D_{\mathrm{PO}}(j \omega)$ as $N_{\mathrm{PE}}, N_{\mathrm{PO}}, D_{\mathrm{PE}}$, and $D_{\mathrm{PO}}$, where $N_{\mathrm{PE}}, N_{\mathrm{PO}}$ are the real part and the imaginary part and $D_{\mathrm{PE}}, D_{\mathrm{PO}}$ are the real part and the imaginary part, respectively. The expressions are given as

$$
\begin{aligned}
& N_{\mathrm{PE}}=\sum_{i=0}^{n} b_{i} \omega^{\beta_{i}} \cos \left(\frac{\beta_{i}}{2} \pi\right) ; \\
& N_{\mathrm{PO}}=\sum_{i=0}^{n} b_{i} \omega^{\beta_{i}} \sin \left(\frac{\beta_{i}}{2} \pi\right), \\
& D_{\mathrm{PE}}=\sum_{i=0}^{n} a_{i} \omega^{\alpha_{i}} \cos \left(\frac{\alpha_{i}}{2} \pi\right) ; \\
& D_{\mathrm{PO}}=\sum_{i=0}^{n} a_{i} \omega^{\alpha_{i}} \cos \left(\frac{\alpha_{i}}{2} \pi\right) .
\end{aligned}
$$

As shown in Figure 1, the internal loop negative feedback transfer function is

$$
\phi_{1}(s)=\frac{G_{p}(s)}{1+C_{\mathrm{PD}}(s) G_{p}(s)} .
$$

The closed-loop characteristic polynomial of the internal loop negative feedback transfer function is written as

$$
\begin{aligned}
\Delta_{\mathrm{PD}}\left(s ; k_{\mathrm{f}}, k_{\mathrm{d}}\right)= & 1+C_{\mathrm{PD}}(s) G_{p}(s) \\
= & (s+100) D_{p}(s)+\left(\left(k_{\mathrm{f}}+100 k_{\mathrm{d}}\right) s+100 k_{\mathrm{f}}\right) \\
& \cdot N_{p}(s) e^{-\tau s} .
\end{aligned}
$$

Substitute $s=j \omega$ and $e^{-j \tau \omega}=\cos (\tau \omega)-j \sin (\tau \omega)$ into inner-loop transfer function (8) and divide it into real part and imaginary part as follows:

$$
\begin{aligned}
\Delta_{\mathrm{PD}}\left(j \omega ; k_{\mathrm{f}}, k_{\mathrm{d}}\right)= & 100\left(D_{\mathrm{PE}}+j D_{\mathrm{PO}}\right)+\omega\left(j D_{\mathrm{PE}}-D_{\mathrm{PO}}\right) \\
& +\left(\left(m_{1} N_{\mathrm{PE}}+m_{2} N_{\mathrm{PO}}\right)+j\left(m_{1} N_{\mathrm{PO}}\right.\right. \\
& \left.\left.+m_{2} N_{\mathrm{PE}}\right)\right) \cos (\tau \omega) \\
& +\left(\left(m_{1} N_{\mathrm{PO}}+m_{2} N_{\mathrm{PE}}\right)-j\left(m_{1} N_{\mathrm{PE}}\right.\right. \\
& \left.\left.+m_{2} N_{\mathrm{PO}}\right)\right) \sin (\tau \omega) \\
= & \operatorname{Re}_{\Delta, \mathrm{PD}}+j \operatorname{Im}_{\Delta, \mathrm{PD}}=0,
\end{aligned}
$$

where $m_{1}=100 k_{\mathrm{f}}$ and $m_{2}=\left(k_{\mathrm{f}}+100 k_{\mathrm{d}}\right) \omega$.

Taking the real part and the imaginary part of the characteristic polynomial as zero, they can be simplified into the following form:

$$
\left\{\begin{array}{l}
\operatorname{Re}_{\Delta, \mathrm{PD}}=A_{1} k_{\mathrm{f}}+B_{1} k_{\mathrm{d}}+C_{1}=0, \\
\operatorname{Im}_{\Delta, \mathrm{PD}}=A_{2} k_{\mathrm{f}}+B_{2} k_{\mathrm{d}}+C_{2}=0,
\end{array}\right.
$$

where $A_{1}=\left(100 N_{\mathrm{PE}}+\omega N_{\mathrm{PO}}\right) \cos (\tau \omega)+\left(100 N_{\mathrm{PO}}+\omega N_{\mathrm{PE}}\right) \sin$ $(\tau \omega) ; \quad A_{2}=\left(100 N_{\mathrm{PO}}+\omega N_{\mathrm{PE}}\right) \cos (\tau \omega)-\left(100 N_{\mathrm{PE}}+\omega N_{\mathrm{PO}}\right)$ $\sin (\tau \omega) ; \quad B_{1}=100 \omega N_{\mathrm{PO}} \cos (\tau \omega)+100 \omega N_{\mathrm{PE}} \sin (\tau \omega) ; \quad B_{2}=$ $100 \omega N_{\mathrm{PE}} \cos (\tau \omega)-100 \omega N_{\mathrm{PO}} \sin (\tau \omega) ; C_{1}=100 D_{\mathrm{PE}}-\omega D_{\mathrm{PO}}$; and $C_{2}=100 D_{\mathrm{PO}}-\omega D_{\mathrm{PE}}$.

Solving equation (10), we can get the expression of $k_{\mathrm{f}}$ and $k_{\mathrm{d}}$ and draw the stable boundary curve on the $k_{\mathrm{d}}-k_{\mathrm{f}}$ plane when $\omega \in(0, \infty)$. The expressions $k_{\mathrm{f}}$ and $k_{\mathrm{d}}$ are given

$$
\left\{\begin{array}{l}
k_{\mathrm{d}}=\frac{A_{1} C_{2}-A_{2} C_{1}}{B_{1} A_{2}-A_{1} B_{2}}, \\
k_{\mathrm{f}}=\frac{B_{2} C_{1}-B_{1} C_{2}}{B_{1} A_{2}-A_{1} B_{2}} .
\end{array}\right.
$$

According to the obtained SBL, the vertices and corner points of the stable region are devoted to describe the CSR. In a general $k_{\mathrm{d}}-k_{\mathrm{f}}$ plane, the CSR is designed as a triangle with one vertex and two corner points, whose coordinates are $\left(k_{\mathrm{d} 1}, k_{\mathrm{f} 1}\right),\left(k_{\mathrm{d} 2}, k_{\mathrm{f} 2}\right)$, and $\left(k_{\mathrm{d} 3}, k_{\mathrm{f} 3}\right)$. In order to ensure that the obtained point is in the stable region, here we propose the concept of the incenter of the CSR, whose coordinate expression is as follows:

$$
H=\frac{\left[\begin{array}{lll}
k_{\mathrm{d} 1} & k_{\mathrm{d} 2} & k_{\mathrm{d} 3} \\
k_{\mathrm{f} 1} & k_{\mathrm{f} 2} & k_{\mathrm{f} 3}
\end{array}\right]\left[\begin{array}{l}
a \\
b \\
c
\end{array}\right]}{a+b+c},
$$

where $a, b$, and $c$ are the modules of the three-sided vectors. The vector $H$ obtained in (12) is the inner-loop PD controller parameter $\left(k_{\mathrm{d}} ; k_{\mathrm{f}}\right)$.

Then, the inner-loop negative feedback system has the following form:

$$
\begin{aligned}
\phi_{1}(s) & =\frac{N(s)}{D(s)}=\frac{G_{p}(s)}{1+C_{\mathrm{PD}}(s) G_{p}(s)} \\
& =\frac{(s+100) N_{p}(s) e^{-\tau s}}{(s+100) D_{p}(s)+N_{p}(s) e^{-\tau s}\left(\left(k_{\mathrm{f}}+100 k_{\mathrm{d}}\right) s+100 k_{\mathrm{f}}\right)} .
\end{aligned}
$$


Simplify the inner-loop transfer function (13) and split the numerator and denominator into real parts and imaginary parts, respectively:

$$
\frac{N(j \omega)}{D(j \omega)}=\frac{N_{E}(j \omega)+j N_{O}(j \omega)}{D_{E}(j \omega)+j D_{O}(j \omega)} .
$$

Simplify $N_{E}(j \omega), N_{O}(j \omega), D_{E}(j \omega)$, and $D_{O}(j \omega)$ as $N_{E}$, $N_{O}, D_{E}$, and $D_{O}$, where $N_{E}, N_{O}$ are the real part and imaginary part of numerator and $D_{E}, D_{O}$ are the real part and imaginary part of the denominator, respectively. Their expressions are as follows:

$$
\begin{aligned}
N_{E}= & \left(N_{\mathrm{PE}} \cos (\tau \omega)+N_{\mathrm{PO}} \sin (\tau \omega)\right)\left(n_{1}+100\right)+\left(N_{\mathrm{PE}} \sin (\tau \omega)\right. \\
& \left.-N_{\mathrm{PO}} \cos (\tau \omega)\right) n_{2} \\
N_{O}= & \left(N_{\mathrm{PE}} \cos (\tau \omega)+N_{\mathrm{PO}} \sin (\tau \omega)\right) n_{2}+\left(N_{\mathrm{PO}} \cos (\tau \omega)\right. \\
& \left.-N_{\mathrm{PE}} \sin (\tau \omega)\right)\left(n_{1}+100\right) \\
D_{E}= & A_{1} k_{\mathrm{f}}+B_{1} k_{\mathrm{d}}+C_{1} \\
D_{\mathrm{O}}= & A_{2} k_{\mathrm{f}}+B_{2} k_{\mathrm{d}}+C_{2} .
\end{aligned}
$$

Regarding the inner loop as a whole, the transfer function of the system is written as

$$
\phi_{2}(s)=\frac{C_{\mathrm{PI}}(s) \phi_{1}(s)}{1+C_{\mathrm{PI}}(s) \phi_{1}(s)} .
$$

Therefore, the closed-loop characteristic equation of the outer loop is determined as follows:

$$
\begin{aligned}
\Delta_{\mathrm{PI}}\left(s ; k_{\mathrm{p}}, k_{\mathrm{i}}\right) & =1+C_{\mathrm{PI}}(s) G_{p}(s) \\
& =s D(s)+\left(\left(k_{\mathrm{p}} s+k_{\mathrm{d}}\right) N(s)\right) .
\end{aligned}
$$

Substitute (4) and (12) into the closed-loop characteristic equation (16) and divide it into real part and imaginary parts:

$$
\Delta_{\mathrm{PI}}\left(j \omega ; k_{\mathrm{p}}, k_{\mathrm{i}}\right)=\operatorname{Re}_{\Delta, \mathrm{PI}}+j \operatorname{Im}_{\Delta, \mathrm{PI}}=0 .
$$

Simplify the characteristic equation into a binary equations with unknown parameters $k_{\mathrm{p}}, k_{\mathrm{i}}$ :

$$
\left\{\begin{array}{l}
\operatorname{Re}_{\Delta, \mathrm{PI}}=\overline{A_{1}} k_{\mathrm{p}}+\overline{B_{1}} k_{\mathrm{i}}+\overline{C_{1}}=0, \\
\operatorname{Im}_{\Delta, \mathrm{PI}}=\overline{A_{2}} k_{\mathrm{p}}+\overline{B_{2}} k_{\mathrm{i}}+\overline{C_{2}}=0,
\end{array}\right.
$$

where $\overline{A_{1}}=-\omega N_{O} ; \quad \overline{A_{2}}=\omega N_{E} ; \quad \overline{B_{1}}=N_{E} ; \quad \overline{B_{2}}=N_{O}$; $\overline{C_{1}}=-\omega D_{O}$; and $\overline{C_{2}}=\omega D_{E}$.

The parameters of SBL and the stability region of the outer-loop PI controller on the $k_{\mathrm{p}}-k_{\mathrm{i}}$ plane are obtained by solving equation (19). And a SBL is drawn on the $k_{\mathrm{p}}-k_{\mathrm{i}}$ plane:

$$
\left\{\begin{array}{c}
k_{\mathrm{p}}=\frac{\overline{B_{2}} \overline{C_{1}}-\overline{B_{1}} \overline{C_{2}} \overline{A_{2}}-\overline{A_{1}} \overline{B_{2}}}{} \\
k_{\mathrm{i}}=\frac{\overline{A_{1}} \overline{C_{2}}-\overline{A_{2}} \overline{C_{1}}}{\overline{B_{1}} \overline{A_{2}}-\overline{A_{1}} \overline{B_{2}}} .
\end{array}\right.
$$

For obtaining the outer-loop PI controller parameters, this paper introduces a Fermat point concept that has the smallest sum of distances to the vertices of the polygon. In order to optimize calculation of Fermat point, a trapezoid is designed at first. The four vertices include two corner points and two points on the SBL which are 1/2 of the longitudinal distance between the cusp and corner point. Fermat point is the diagonal intersection point.

The proposed method includes the following five steps:

Step 1: according to equation (11), the relationship between the parameters $k_{\mathrm{d}}, k_{\mathrm{f}}$ of the inner-loop PD controller could be obtained. And the SBL could be drawn on the $k_{\mathrm{d}}-k_{\mathrm{f}}$ plane.

Step 2: by selecting the vertices and corner points of the CSR, the incenter of the convex CSR is computed according to equation (12). Its coordinates $H\left(k_{\mathrm{d}}, k_{\mathrm{f}}\right)$ are the parameters of the inner-loop PD controller.

Step 3: calculating the transfer function of the inner loop based on the obtained parameters $\left(k_{\mathrm{d}}, k_{\mathrm{f}}\right)$.

Step 4: according to equation (20), the relation of the outer-loop PI controller parameters $k_{\mathrm{p}}, k_{\mathrm{i}}$ could be obtained. The SBL is drawn on the $k_{\mathrm{p}}-k_{\mathrm{i}}$ plane.

Step 5: determining the corner point and the boundary point of the SBL; the intersection point of the trapezoid diagonal is the parameter of the outer-loop PI controller.

Remark 1. For fractional-order time-delay systems, the traditional method of selecting stable regions is usually determined by manually selecting a large number of test points in all the regions divided by the stable boundary trajectories, which will lead to time consumption. Inspired by [37], this paper gives a simple parameter tuning method of FOPID controller, which avoids time-consuming stability test.

2.3. Method Extension of Uncertain Systems. Due to modelling errors and other factors, it is an important issue for the analysis and design of the uncertain parameter control systems with time delay in control theory. In this article, the design method is extended to the fractional-order system with time delay and parameter uncertainty. Multiple transfer functions are attained in the condition of uncertain parameters. Consider the transfer function with parameter uncertainty for the control system shown in Figure 1. It is defined as follows:

$$
G_{p}(s)=\frac{\sum_{j=0}^{n} b_{j} s^{\beta_{j}}}{\sum_{i=0}^{m} a_{i} s^{\alpha_{i}}} e^{-\tau s},
$$

where $a_{i} \in\left[\underline{a}_{i}, \bar{a}_{i}\right], b_{j} \in\left[\underline{b}_{j}, \bar{b}_{j}\right], \alpha_{i} \in\left[\underline{\alpha}_{i}, \bar{\alpha}_{i}\right], \beta_{j} \in\left[\underline{\beta}_{j}, \bar{\beta}_{j}\right], i=$ $0,1, \ldots, m$ and $j=0,1, \ldots, n$. According to the number of uncertain parameters in the general transfer function, there are $m^{2} \times n^{2}$ Kharitonov plants in total.

For the uncertain parameter system with time delay, all steps of the method put forward in paper are individually processed for each transfer function in terms of the Kharitonov theorem. Then, the common stable domain of all transfer functions and its convex stable domain are obtained. 
Finally, the incenter of the inner loop and the Fermat points of outer loop of the common convex stability region are obtained, and then a robust PI-PD controller is designed.

The proposed method extension of the time-delay uncertain system could be classified into the following steps:

Step 1: for all submodels, the inner-loop stable boundary loci are calculated separately, and the common stable region is determined graphically on the $k_{\mathrm{d}}-$ $k_{\mathrm{f}}$ plane.

Step 2: then, the CCSR is determined according to the corner points, intersection points, and individual tip points. The incenter of the CCSR is computed by applying (12). The coordinate of the incenter is the parameter $\left(k_{\mathrm{d}}, k_{\mathrm{f}}\right)$ of the $\mathrm{PD}$ controller in the internal loop.

Step 3: by substituting the values $k_{\mathrm{d}}$ and $k_{\mathrm{f}}$ into the inner-loop system, the transfer functions of the outer loop are obtained for all the submodels simplified based on the Kharitonov theorem.

Step 4: the common stable region of the outer-loop PI controller on the $k_{\mathrm{p}}-k_{\mathrm{i}}$ plane is determined based on all closed-loop transfer functions.

Step 5: looking for corner points, tip points, and bipartite points of the common stable area, the CCSR based on these points is obtained. The Fermat point of the CCSR is given by the graphical method, which is parameter $\left(k_{\mathrm{p}}, k_{\mathrm{i}}\right)$ of the outer-loop PI controller.

Remark 2. The method proposed in this paper is an empirical parameter adjustment method, which is only suitable for systems that can clearly distinguish stable regions in images. For uncertain systems, it is only applicable to the case with common stable region. Otherwise, further research is needed to find other control methods.

\section{Simulation}

Several simulation examples show the effectiveness of the proposed method. It includes the following plants: two unstable time-delay systems of order 1 and order 2, respectively, the time-delay unstable fractional plant with uncertain parameters, and the two fractional-order plants.

3.1. Example A. Consider a first-order unstable transfer function with time delay which was studied by Tan [29], Onat [34], Kaya [38], and Visioli [39]. It has the following form:

$$
G_{p}(s)=\frac{4}{4 s-1} e^{-2 s}
$$

According to the proposed steps, the stable region of the inner-loop system is obtained. The SBL is drawn in the $k_{\mathrm{d}}-k_{\mathrm{f}}$ plane. And the convex region of the stable region is demonstrated in Figure 2. It shows that the convex stability region consists of one vertex $V$ and two corner points $C_{1}$ and $C_{2}$. The convex stability region of the $\mathrm{PD}$ controller can be described as a triangle by these points. According to the coordinates of the above points, the incenter (Inc) of the PD controller can be calculated by applying equation (12), and its coordinate is (0.461, $0.469)$. The inner-loop PD controller parameters are $k_{\mathrm{d}}=0.461$ and $k_{\mathrm{f}}=0.499$.

Substituting the PD controller parameters into the inner loop, the system transfer function is obtained by using (20). For the outer loop, PI controller parameters' stability region is drawn in the $k_{\mathrm{p}}-k_{\mathrm{i}}$ plane in Figure 3. Figure 3 also demonstrates the CSR. In this step, two corner points $C_{3}$ and $C_{4}$ and two points $E_{1}$ and $E_{2}$ on the SBL with a longitudinal distance of $1 / 2$ from the corner point to the cusp point are given as shown in Figure 3. These points describe the convex region as a trapezoid. Then, the coordinates of Fermat points (Fps) are obtained as $(0.06475,0.04573)$, and the parameters of the inner-loop PI controller are $k_{\mathrm{p}}=0.06459$ and $k_{\mathrm{i}}=0.04564$. Thus, parameters of the PI-PD controller are $k_{\mathrm{d}}=$ $0.461, k_{\mathrm{f}}=0.499, k_{\mathrm{p}}=0.06459$, and $k_{\mathrm{i}}=0.04564$.

In Figure 4, the unit step response of the method put forward in this paper is compared with the methods put forward by Onat [34], Tan [29], Kaya [38], and Visioli [39]. Table 1 gives the parameters of the various methods. The settling time, overshoot, rise time, peak time, and IAE values are used as evaluation index. Table 2 gives the evaluation values of this proposed method, other controller designs, and optimization methods. In light of the evaluation criteria, the tuning method proposed in this paper exhibits the superior performance. This method has the lowest settling time and the smallest overshoot. The response speed of the PID controller is fast, but other performances are not ideal. In this example, the PI-PD controller proved to be superior to PID controller, and the parameter tuning method is the best in comparison.

3.2. Example B. View a second-order unstable transfer function which was studied by Kaya and Atherton [28]:

$$
G_{p}(s)=\frac{1}{2 s^{2}+s-1} e^{-0.3 s} .
$$

The stability region of the inner-loop PD controller is shown in Figure 5. A vertex $V$ and two corner points $C_{1}$ and $C_{2}$ in Figure 5 construct a CSR. Inc stands for convex stable region, and Inc can be calculated as $(5.51,4.8667)$, and then the inner-loop PD controller parameters are $k_{\mathrm{d}}=5.51$ and $k_{\mathrm{f}}=4.8667$.

By substituting the PD controller parameters $\left(k_{\mathrm{d}}=5.51, k_{\mathrm{f}}=4.8667\right)$ into the inner loop, the transfer function can be obtained by using (12). Figure 6 gives the stable region and its convex region of the outer ring PI controller in the $k_{\mathrm{p}}-k_{\mathrm{i}}$ plane. $C_{3}$ and $C_{4}$ are the corner points and $E_{1}$ and $E_{2}$ are the bisection points. The diagonal intersection coordinate of the quadrilateral is obtained by the graphic method, and the coordinate values of $\mathrm{Fp}$ are $k_{\mathrm{p}}=3.77$ and $k_{\mathrm{i}}=4.75$. Through the above analysis and calculation, the PI-PD controller parameters calculated by 


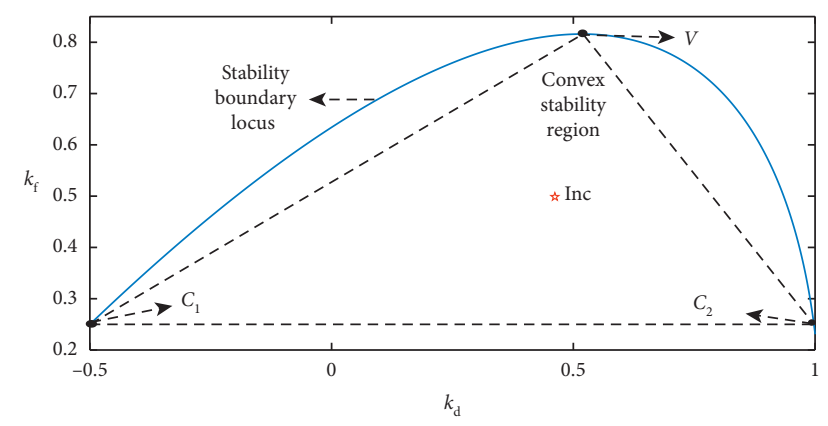

FIGURE 2: Convex stability region and incenter of PD controller in example A.

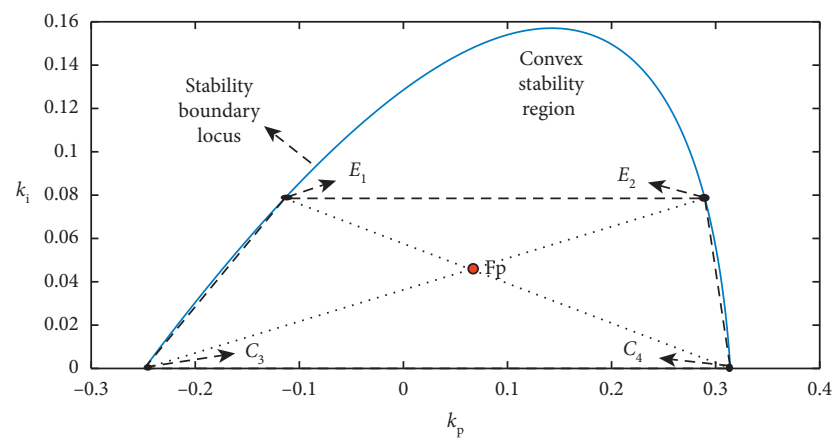

FIgURE 3: Convex stability region and Fermat point of PI controller in example A.

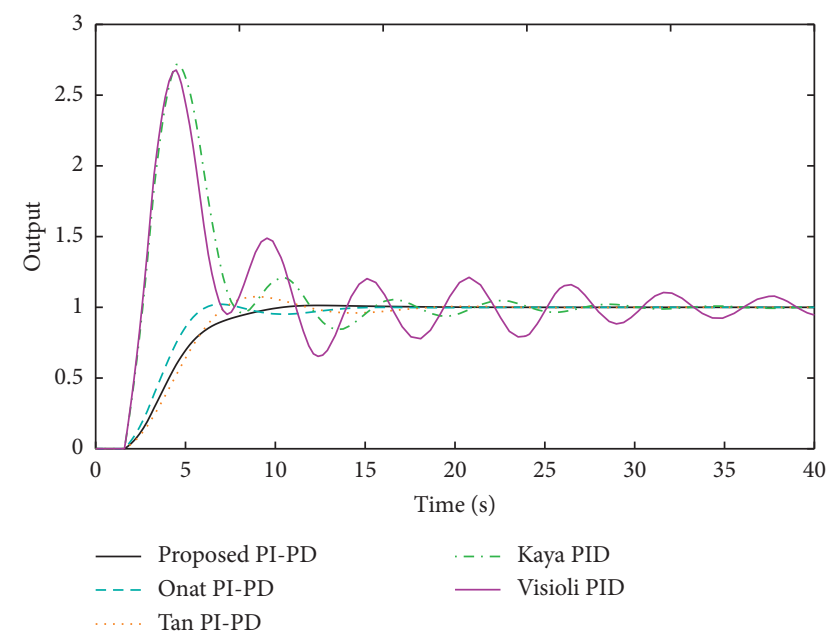

FIGURE 4: Unit step responses comparison of various methods for example A.

the proposed method are determined. They are $k_{\mathrm{d}}=5.51$, $k_{\mathrm{f}}=4.8667, k_{\mathrm{p}}=3.77$, and $k_{\mathrm{i}}=4.75$.

Figure 7 gives the comparison between the PI-PD controller optimized by the parameter tuning method put forward in this paper and the controller put forward by Kaya and Atherton [28]. The unit step responses of these controllers are illustrated in Figure 7. Controller parameters are shown in Table 3. Table 4 gives the performance indexes of the several studies in this example. Obviously, the proposed method has lowest overshoot and peak time. And it also has a small IAE in this example. It is obvious that the tuning method put forward in this paper is better than the others.

3.3. Example C. This example considers a time-delay unstable transfer function with uncertain parameters. The transfer function is written as

$$
G_{p}(s)=\frac{1}{s^{a}-b} e^{-0.2 s},
$$

where $a \in[0.8,1.2]$ and $b \in[0.5,1.5]$. Four boundary transfer functions are taken into account based on Kharitonov's theorem which are $G_{p 1}(s), G_{p 2}(s), G_{p 3}(s)$, and $G_{p 4}(s)$. The plants are given in (25)-(28), respectively:

$$
\begin{aligned}
& G_{p 1}(s)=\frac{1}{s^{1.2}-0.5} e^{-0.2 s}, \\
& G_{p 2}(s)=\frac{1}{s^{1.2}-1.5} e^{-0.2 s}, \\
& G_{p 3}(s)=\frac{1}{s^{0.8}-1.5} e^{-0.2 s}, \\
& G_{p 4}(s)=\frac{1}{s^{0.8}-0.5} e^{-0.2 s} .
\end{aligned}
$$

Above all, stability boundary loci of edge plants in the inner loop are drawn in the $k_{\mathrm{d}}-k_{\mathrm{f}}$ plane in Figure 8 . According to the stable boundary loci obtained above, the common stability region is obtained as shown as the shadow in Figure 8. Figure 9 enlarges CSR in Figure 8 and gives CCSR composed of corner points $C_{1}$ and $C_{2}$ and vertex $V . C_{1}$ is the intersection of two stable boundary loci. The parameters of the inner-loop PD controller are the values of Inc coordinates with $k_{\mathrm{d}}=0.0314$ and $k_{\mathrm{f}}=2.205$.

Furthermore, for outer loop, stability boundary loci of edge plants are drawn in Figure 10, and the shadow is the CSR. Figure 11 enlarges CSR in Figure 10 and gives CCSR as a trapezoid composed of corner points $C_{3}$ and $C_{4}$ and the bisection points $E_{1}$ and $E_{2}$ of the common stable region. Applying the method proposed in the paper, the common convex region is designed as a trapezoid and Fermat point is the diagonal intersection of the trapezoid. Therefore, the values of $\mathrm{Fp}$ coordinate in Figure 11 are the parameters of the outer-loop PI controller with $k_{\mathrm{p}}=1.518$ and $k_{\mathrm{f}}=1.644$. Through the above two steps, the PI-PD controller of the system is obtained, and its parameters are $k_{\mathrm{d}}=0.0314$, $k_{\mathrm{f}}=2.205, k_{\mathrm{p}}=1.518$, and $k_{\mathrm{f}}=1.644$.

Finally, for the parameters of the uncertain system, the cell step responses of the four boundary plants are obtained in Figure 12. For the uncertain system, the controller has strong robustness.

3.4. Example D. Consider the following transfer function, which has an order of 0.8 : 
TABLE 1: Controller parameters comparison of various methods in example A (other data cited from [34]).

\begin{tabular}{|c|c|c|c|c|c|}
\hline Controller & Proposed PI-PD & Onat PI-PD & Tan PI-PD & Kaya PI-PD & Visioli PID \\
\hline Parameters & $\begin{array}{l}k_{\mathrm{d}}=0.461 \\
k_{\mathrm{f}}=0.499 \\
k_{\mathrm{p}}=0.06459 \\
k_{\mathrm{i}}=0.04564\end{array}$ & $\begin{array}{l}k_{\mathrm{d}}=0.3412 \\
k_{\mathrm{f}}=0.439 \\
k_{\mathrm{p}}=0.107 \\
k_{\mathrm{i}}=0.0393\end{array}$ & $\begin{array}{l}k_{\mathrm{d}}=0.20 \\
k_{\mathrm{f}}=0.413 \\
k_{\mathrm{p}}=0.07 \\
k_{\mathrm{i}}=0.03\end{array}$ & $\begin{array}{l}k_{\mathrm{p}}=0.6217 \\
k_{\mathrm{i}}=0.0722 \\
k_{\mathrm{d}}=0.5352\end{array}$ & $\begin{array}{l}k_{\mathrm{p}}=0.652 \\
k_{\mathrm{i}}=0.0789 \\
k_{\mathrm{d}}=0.6309\end{array}$ \\
\hline
\end{tabular}

TABLE 2: Performance metrics comparison of various methods in example A (other data cited from [34]).

\begin{tabular}{lcccr}
\hline Controller & Settling time $(\mathrm{s})$ & Overshoot $(\%)$ & Rise time $(\mathrm{s})$ & Peak time $(\mathrm{s})$ \\
\hline Proposed PI-PD & 11.81 & 1.35 & 5.80 & 15.68 \\
Onat PI-PD & 16.04 & 2.13 & 3.77 & 8.63 \\
Tan PI-PD & 20.26 & 6.24 & 4.64 & 10.62 \\
Kaya PI-PD & 17.70 & 174.16 & 0.45 & 5.87 \\
Visioli PID & 24.15 & 191.65 & 0.32 & 5.89 \\
\hline
\end{tabular}

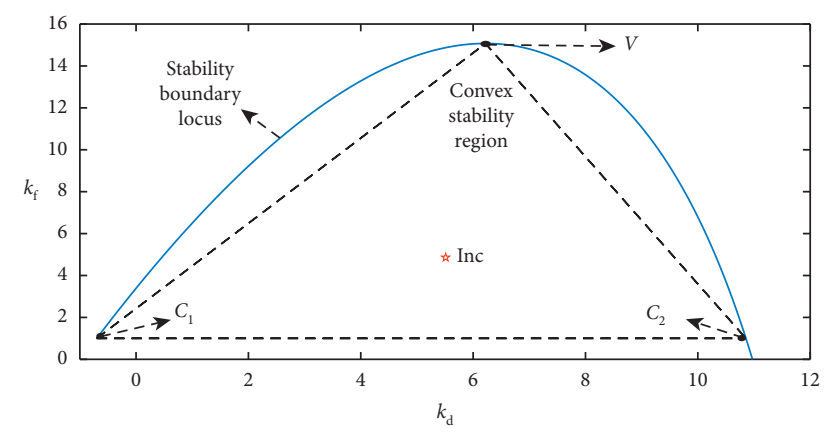

FIGURE 5: Convex stability region and incenter of PD controller in example B.

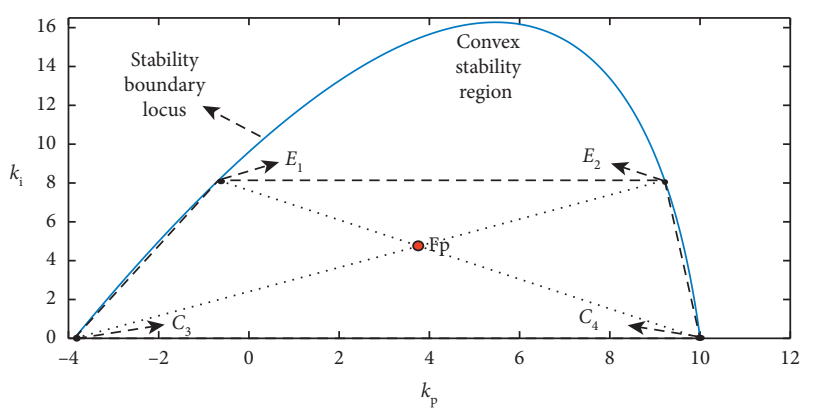

FIGURE 6: Convex stability region and Fermat point of PI controller in example $B$.

$$
G_{p}(s)=\frac{1}{s^{0.8}-1} e^{-0.2 s}
$$

In this example, if the point at $\omega=0$ is used as a corner point, the CSR is as shown in Figure 13. The convex region contains a lot of unstable regions, and the WGC point and the incenter of the convex region are outside of the stable region. Therefore, as shown in Figure 14, a new point $C_{1}$ is defined as the left corner point. The height of $C_{1}$ is equal to the original corner point. And $C_{1}$ is closer to the stable region. $C_{2}$ is another corner point of the new convex stable region, and $V$ is the vertex of the stable region.

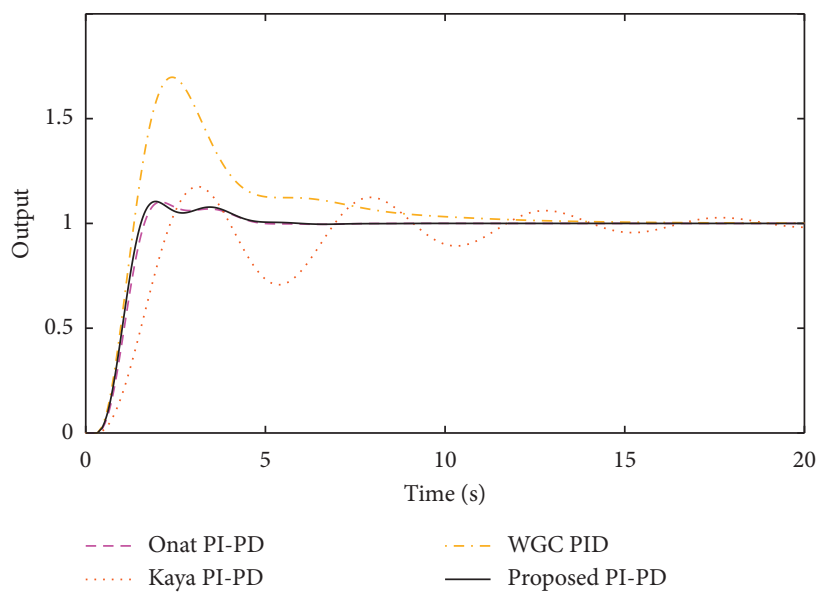

Figure 7: Step responses comparison of various methods for example B.

For inner loop, Inc coordinates are obtained as $k_{\mathrm{d}}=$ -0.041 and $k_{\mathrm{f}}=1.55$ and centroid coordinates are obtained as $k_{\mathrm{d}}=0.00947$ and $k_{\mathrm{f}}=2.5633$.

And for the outer loop, using the WGC method, the convex stability region is given as Figure $15 . C_{3}, C_{4}$, and $C_{5}$ are the corner points of the stable region, and $V$ is the vertex of the stable region. The centroid PI controller parameters are obtained as $k_{\mathrm{p}}=0.1072$ and $k_{\mathrm{i}}=2.805$. Applying the method put forward in this paper, the convex stability region of PI controller is given in Figure 16. The convex stable region is a trapezoid composed of the corner points $C_{6}$ and $C_{7}$ and the bisection points $E_{1}$ and $E_{2}$ of the common stable region. The outer-loop PI controller parameters are computed as Fp with $k_{\mathrm{p}}=1.527$ and $k_{\mathrm{i}}=2.515$. Accordingly, the PI-PD controller optimized by using the WGC method and the method proposed in this paper is determined. The parameters of the WGC method are $k_{\mathrm{d}}=0.00947, k_{\mathrm{f}}=2.5633$, and $k_{\mathrm{p}}=0.1072, k_{\mathrm{i}}=2.805$, and the parameters of the proposed method are $k_{\mathrm{d}}=-0.041, k_{\mathrm{f}}=1.55$, and $k_{\mathrm{p}}=1.527, k_{\mathrm{i}}=2.515$. The unit step responses are given in Figure 17. Compared with Onat's method, the method proposed in this paper can achieve faster response. 
TABLE 3: Controller parameters comparison of various methods in example B (other data cited from [34]).

\begin{tabular}{lllll}
\hline Controller & Proposed PI-PD & Onat PI-PD & Kaya PI-PD & Atherton PID \\
\hline & $k_{\mathrm{d}}=5.51$ & $k_{\mathrm{d}}=5.46$ & $k_{\mathrm{d}}=0.535$ & $k_{\mathrm{p}}=1.398$ \\
Parameters & $k_{\mathrm{f}}=4.8667$ & $k_{\mathrm{f}}=5.69$ & $k_{\mathrm{f}}=3$ & $k_{\mathrm{i}}=0.793$ \\
& $k_{\mathrm{p}}=3.77$ & $k_{\mathrm{p}}=3$ & $k_{\mathrm{p}}=1.398$ & $k_{\mathrm{d}}=0.535$ \\
& $k_{\mathrm{i}}=4.75$ & $k_{\mathrm{i}}=5.3$ & $k_{\mathrm{i}}=0.793$ & \\
\hline
\end{tabular}

TABLE 4: Performance metrics comparison of various methods in example B (other data cited from [34]).

\begin{tabular}{lcccr}
\hline Controller & Settling time $(\mathrm{s})$ & Overshoot $(\%)$ & Rise time (s) & Peak time (s) \\
\hline Proposed PI-PD & 11.81 & 1.35 & 5.80 & 15.68 \\
Onat PI-PD & 16.04 & 2.13 & 3.77 & 8.63 \\
Tan PI-PD & 20.26 & 6.24 & 4.64 & 10.62 \\
Kaya PI-PD & 17.70 & 174.16 & 0.45 & 5.83 \\
Visioli PID & 24.15 & 191.65 & 0.32 & 5.89 \\
\hline
\end{tabular}

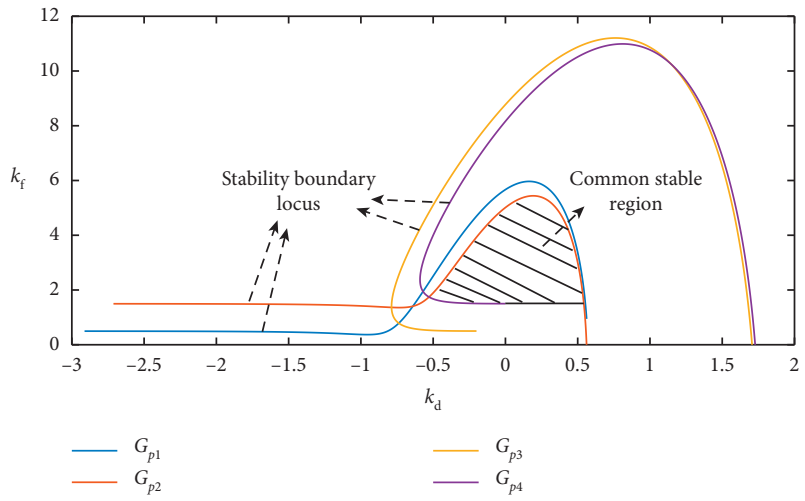

FIGURE 8: Stability boundary loci of inner-loop PD controller for uncertain systems in example C.

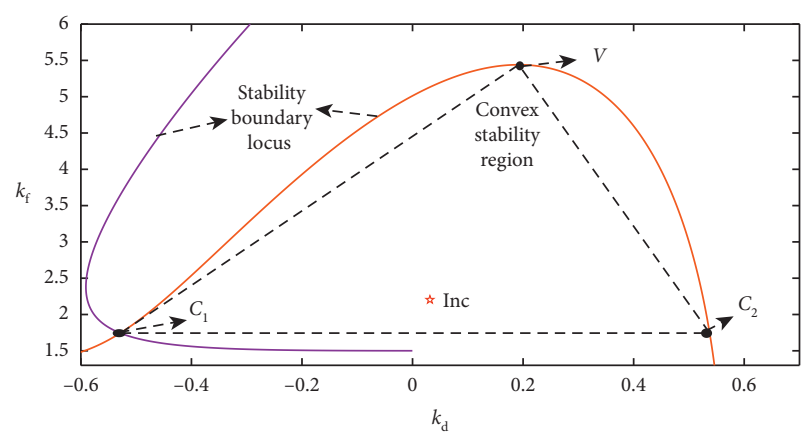

FIGURE 9: Common convex stable region and incenter of PD controller in example C.

3.5. Example E. Consider the case of order 1.2 of fractional transfer function in example 3 :

$$
G_{p}(s)=\frac{1}{s^{1.2}-1} e^{-0.2 s} .
$$

According to the method proposed in this paper, the corner points are selected. The stable region and its regular convex stability region of the inner loop and outer loop are shown in Figures 18 and 19, respectively. $C_{1}, C_{2}, C_{3}$, and $C_{4}$

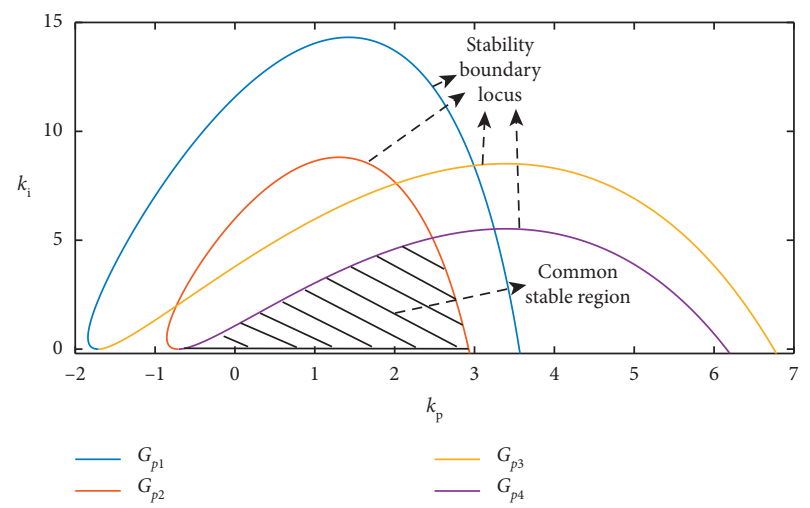

FIGURE 10: Stability boundary loci of outer-loop PI controller for uncertain systems in example C.

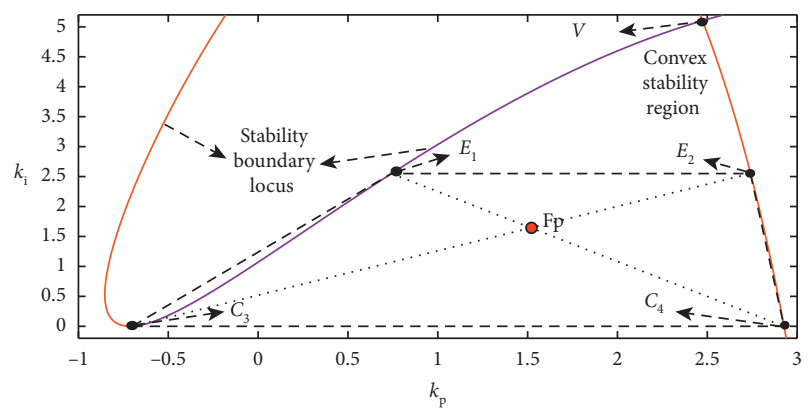

FIgURE 11: Common convex stable region and Fermat point of the PI controller in example C.

are the corner points, $V$ is the vertex point, and $E_{1}$ and $E_{2}$ are the bisection points. The inner PD controller parameters are the coordinates of Inc with $k_{\mathrm{d}}=0.799$ and $k_{\mathrm{f}}=1.823$, and the outer PI controller parameters are the coordinates of Fp with $k_{\mathrm{p}}=4.976$ and $k_{\mathrm{i}}=7.75$.

Due to the inward extension of corner points, the following attempts were made in this paper to select inflection points on the boundary to replace corner points. The stable region and the convex region are as shown in Figures 20 and 


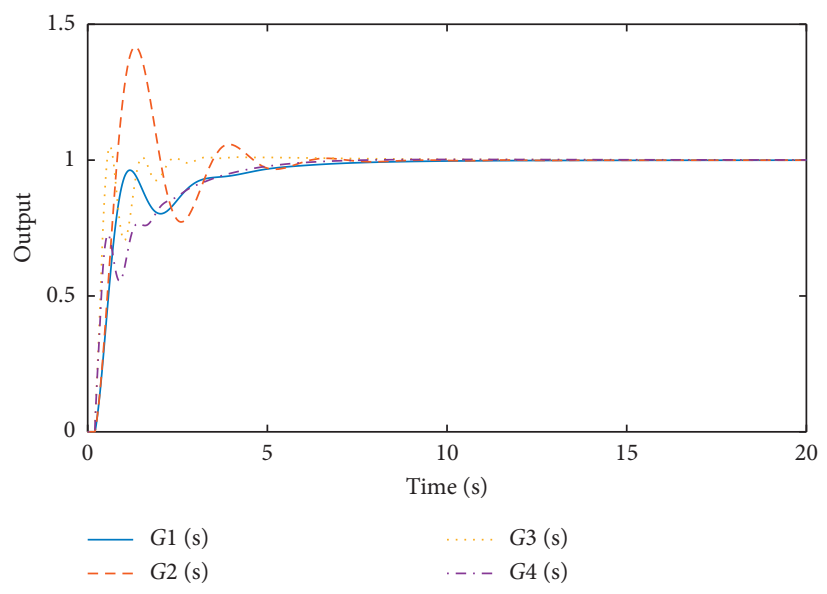

Figure 12: Step response comparison of various methods for example C.

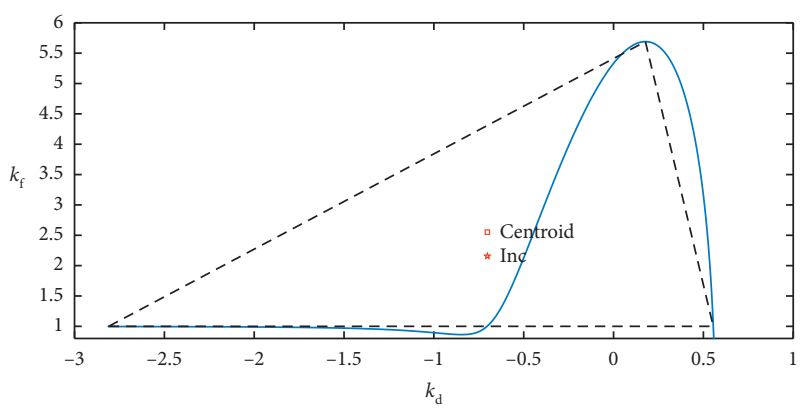

Figure 13: General corner point of inner-loop PD controller in example D.

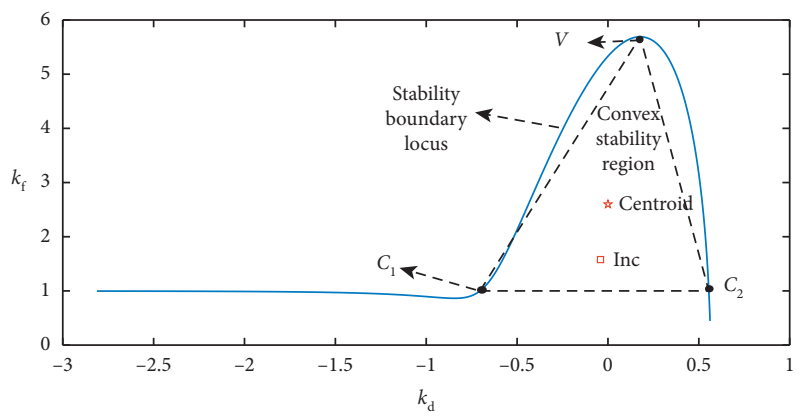

Figure 14: New corner point of inner-loop PD controller in example D.

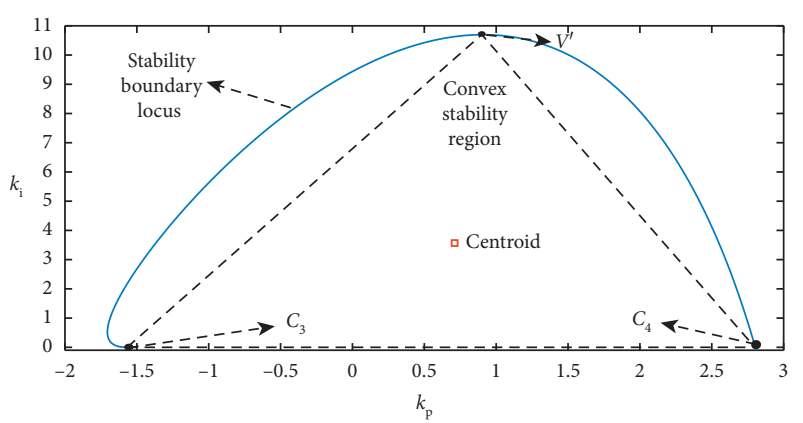

FIGURE 15: Convex stability region and centroid of the PI controller in example D.

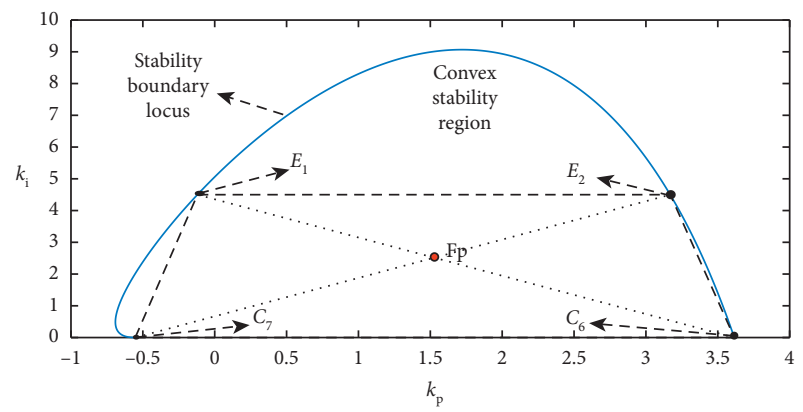

Figure 16: Convex stability region and Fermat point of PI controller in example D.

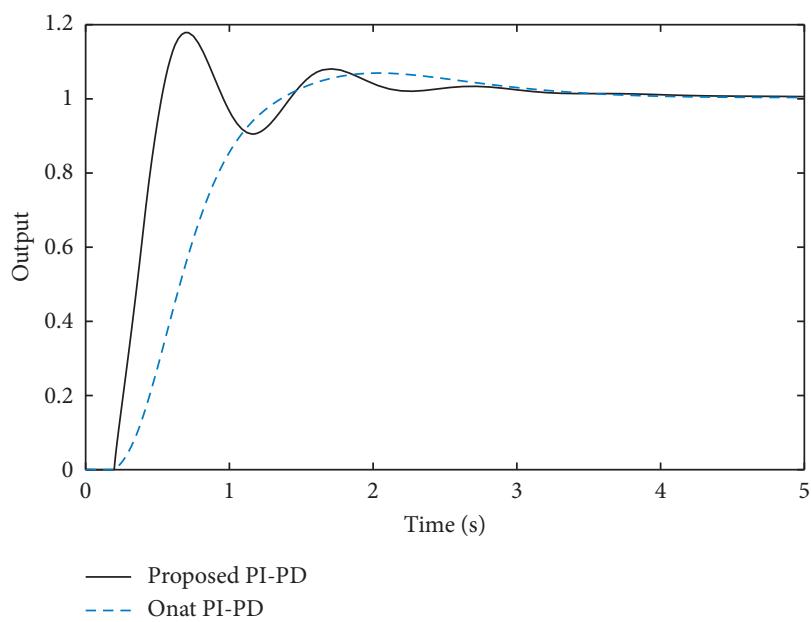

Figure 17: Step responses in example D.

21. $C_{5}, C_{6}, C_{7}$, and $C_{8}$ are the corner points, $V$ is the vertex point, and $E_{3}$ and $E_{4}$ are the bisection points. $\left(k_{\mathrm{d}}, k_{\mathrm{f}}\right)$ and $\left(k_{\mathrm{p}}, k_{\mathrm{i}}\right)$ are the coordinates of Inc and $\mathrm{Fp}$, respectively. The parameters of this improved PI-PD controller are $k_{\mathrm{d}}=0.533, k_{\mathrm{f}}=2.941, k_{\mathrm{p}}=3.488$, and $k_{\mathrm{i}}=5.363$. The unit step responses are compared as shown in Figure 22. The two methods show the same settling time. However, the improved method is better than the former one in the field of overshot and IAE capabilities. 


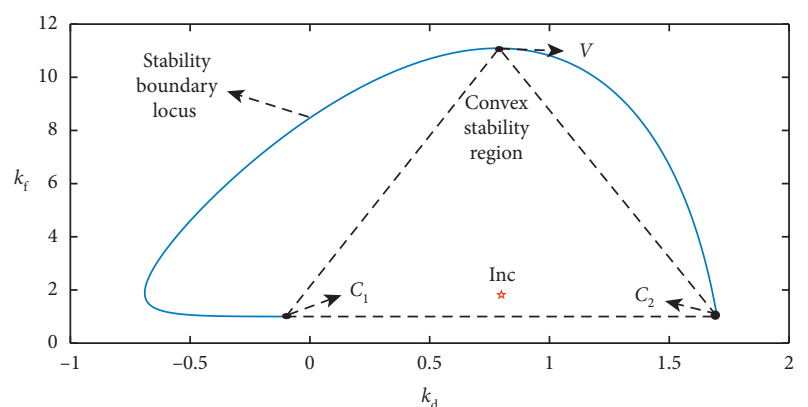

FIgURE 18: Convex stability region designed by regular point in example E.

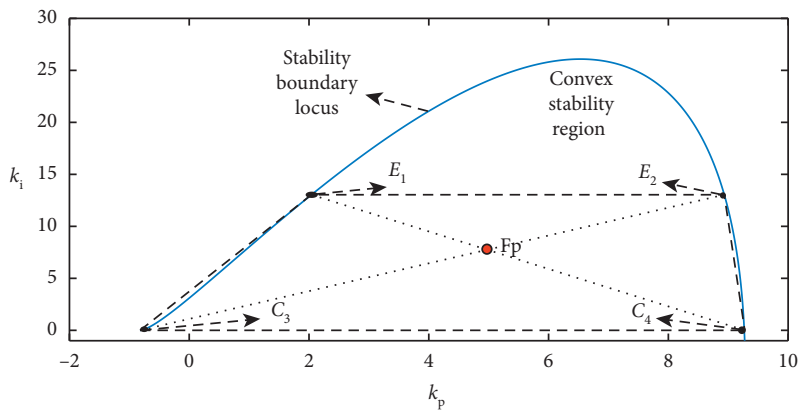

Figure 19: Regular convex stability region and Fermat point of PI controller in example E.

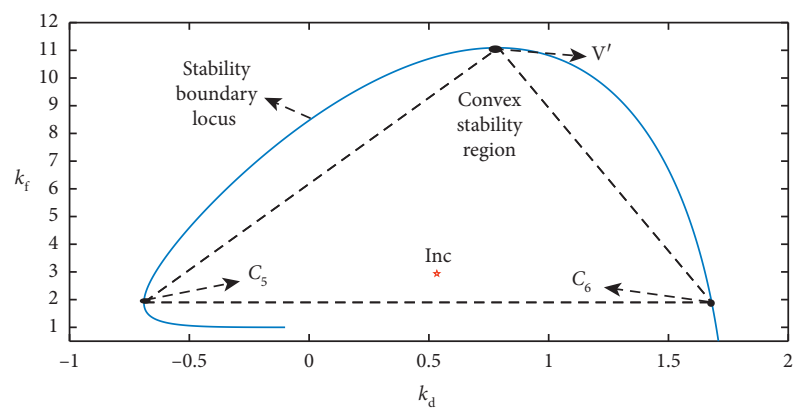

FIgURE 20: Changed convex stability region and incenter of PD controller in example E.

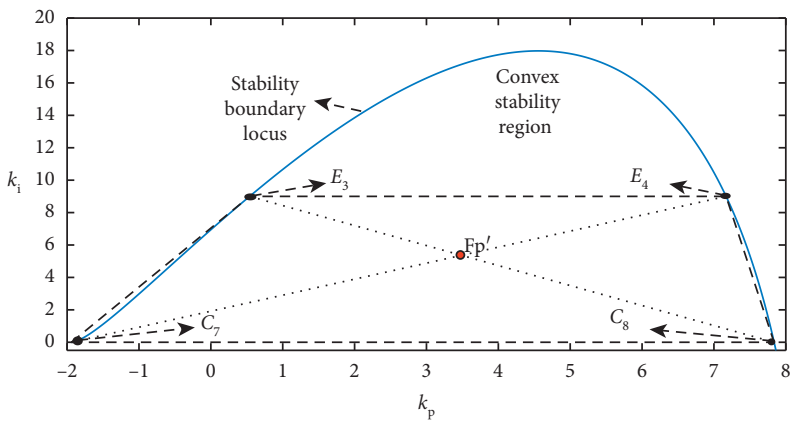

FIGURE 21: Changed convex stability region and Fermat point of PI controller in example E.

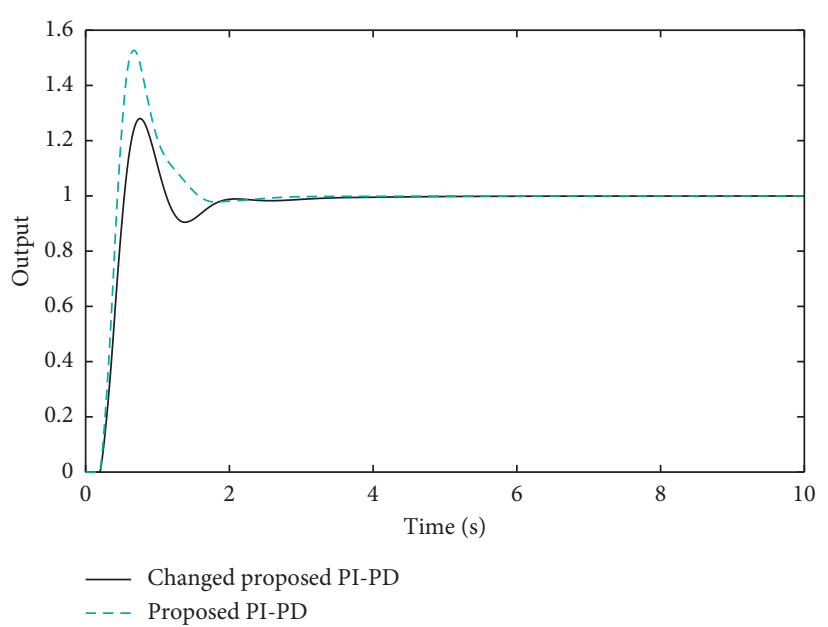

Figure 22: Step response for example E.

\section{Conclusions}

In this paper, a PI-PD control parameter graphical tuning method for unstable fractional time-delay systems is proposed by using the concept of incenter and Fermat points of CSRs. The method obtains the inner-loop stable boundary locus and the outer-loop stable boundary locus by establishing the characteristic equations. CSR is designed, and the incenter of the inner-loop CSR and Fermat point of outerloop convex stable region are obtained, respectively. This method uses several special points on the stable boundary locus to determine the convex stable region. The method has the advantages of convenient calculation and easy implementation in engineering. This method is popularized to the uncertain system by applying Kharitonov's theorem. Simulation examples verify the effectiveness of the method put forward in the paper. In future studies, the proposed method can also be extended to the $\mathrm{PI}^{\lambda}-\mathrm{PD}^{\mu}$ controller.

\section{Nomenclature}

SBL: $\quad$ Stable boundary locus

ISE: Integral of squared error

ISTE: Integral of squared time weighted error

WGC: Weight geometric centre

CSR: Convex stable region

CCSR: Common convex stable region

$k_{\mathrm{d}}$ : $\quad$ Derivative gain of the PD controller

$k_{\mathrm{f}}$ : $\quad$ Proportional gain of the PD controller

$k_{\mathrm{p}}$ : $\quad$ Proportional gain of the PI controller

$k_{\mathrm{i}}$ : $\quad$ Integral gain of the PI controller

$C_{\mathrm{PI}}: \quad$ Transfer function of the PI controller

$C_{\mathrm{PD}}$ : Transfer function of the PD controller

$s: \quad$ Laplace variable

$j: \quad \quad$ Imaginary $\sqrt{-1}$

$\omega: \quad$ Frequency

$\Delta: \quad$ Characteristic equation

$N$ : $\quad$ Numerator of the transfer function

$D: \quad$ Denominator of the transfer function

Inc: Incenter of the convex stable region 
Fp: $\quad$ Fermat point

$C_{1}, C_{2}, \ldots:$ Corner of the stable region

$V: \quad$ Vertex of the stable region

\section{Data Availability}

Processing data applied to support the results of this study are included within the article.

\section{Conflicts of Interest}

The authors declare that they have no conflicts of interest.

\section{Acknowledgments}

This study was supported in part by Shanghai Key Laboratory of Power Station Technology and National Natural Science Foundation under grant no. 61873335.

\section{References}

[1] S. Bouabdallah, A. Noth, and R. Siegwart, "PID vs. LQ control techniques applied to an indoor micro quadrotor," in Proceedings of the 2004 IEEE/RSJ International Conference on Intelligent Robots and Systems, vol. 3, pp. 2451-2456, IEEE, Sendai, Japan, September 2004.

[2] K. S. Tang, K. F. Man, G. R. Chen, and S. Kwong, "An optimal fuzzy PID controller," IEEE Transactions on Industrial Electronics, vol. 48, no. 4, pp. 757-765, 2001.

[3] S. Skogestad, "Simple analytic rules for model reduction and PID controller tuning," Modelling Identification \& Control, vol. 13, no. 4, pp. 291-309, 2004.

[4] K. J. Åström and T. Hägglund, "Revisiting the Ziegler-Nichols step response method for PID control," Journal of Process Control, vol. 14, no. 6, pp. 635-650, 2004.

[5] Y. Lee, S. Park, M. Lee, and C. Brosilow, "PID controller tuning for desired closed-loop responses for SI/SO systems," AIChE Journal, vol. 44, no. 1, pp. 106-115, 1998.

[6] K. H. Ang, G. Chong, and L. Yun, "PID control system analysis, design, and technology," IEEE Transactions on Control Systems Technology, vol. 13, no. 4, pp. 559-576, 2005.

[7] Y. Yi, D. Chen, and Q. Xie, "Controllability of nonlinear fractional order integrodifferential system with input delay," Mathematical Methods in the Applied Sciences, vol. 42, no. 11, pp. 3799-3817, 2019.

[8] I. Kaya, "A PI-PD controller design for control of unstable and integrating processes," ISA Transactions, vol. 42, no. 1, pp. 111-121, 2003.

[9] I. Kaya, "PI-PD controllers for controlling stable processes with inverse response and dead time," Electrical Engineering, vol. 98, no. 1, pp. 55-65, 2016.

[10] E. E. Ezema, I. I. Eneh, and O. L. Daniya, "Improving structural limitations of PID controller for unstable processes," International Journal of Engineering Research \& Applications, vol. 4, no. 9, pp. 87-90, 2014.

[11] Z. Yan, "Research on application of fuzzy PID controller in two-container water tank system control," in Proceedings of the International Conference on Machine Vision \& HumanMachine Interface, April 2010.

[12] K. J. Åström and T. Hägglund, "The future of PID control," Control Engineering Practice, vol. 9, no. 11, pp. 1163-1175, 2001.
[13] S. M. Asyraf, P. M. Heerwan, and I. M. Izhar, "The comparison respond of braking torque control between PID and SMC controller for electric powered wheelchair descending on slope condition," IOP Conference Series: Materials Science \& Engineering Conference Series, vol. 342, Article ID 012088, 2018.

[14] C. Zeng, Develop a Robust Nonlinear Controller for Large Aircraft by Applying NDI, SMC and Adaptive Control, Cranfield University, Cranfield, UK, 2012.

[15] M. Zamani, M. Karimi-Ghartemani, N. Sadati, and M. Parniani, "Design of a fractional order PID controller for an AVR using particle swarm optimization," Control Engineering Practice, vol. 17, no. 12, pp. 1380-1387, 2009.

[16] C. Qin, N. Qi, and Z. Song, "Fractional PID controller design of hypersonic flight vehicle," in Proceedings of the 2010 International Conference on Computer, Mechatronics, Control and Electronic Engineering, August 2010.

[17] P. Roy, S. Sarkar, B. K. Roy, and N. Singh, "A comparative study between fractional order SMC and SMC applied to magnetic levitation system," in Proceedings of the 2017 Indian Control Conference, pp. 473-478, IEEE, Guwahati, India, January 2017.

[18] Z. Jia, X. Sun, and N. Zhang, "A now type of nonnnear controller-nine-point controller," in Proceedings of the 2002 International Conference on Control and Automation (ICCA), Xiamen, China, June 2002.

[19] A. Kumar and V. Kumar, "Evolving an interval type-2 fuzzy PID controller for the redundant robotic manipulator," $E x$ pert Systems with Applications, vol. 73, pp. 161-177, 2017.

[20] S. Nema and P. K. Padhy, "PI-PD controller for stable and unstable processes," International Journal of Systems, Control and Communications, vol. 5, no. 2, pp. 156-165, 2013.

[21] N. K. Vastrakar and P. K. Padhy, "Simplified PSO PI-PD controller for unstable processes," in Proceedings of the 2013 International Conference on Intelligent Systems Modelling \& Simulation, January 2013.

[22] G. A. Hassaan, "Robustness of I-PD, PD-PI and PI-PD controllers used with second-order processes," International Journal of Scientific \& Technical Research, vol. 3, no. 10, 2014.

[23] H. Gao and T. Chen, " $\$\{\backslash \text { cal } H\}_{-}\{\backslash$ infty $\} \$$ estimation for uncertain systems with limited communication capacity," IEEE Transactions on Automatic Control, vol. 52, no. 11, pp. 2070-2084, 2007.

[24] S. Wang, B. Chen, and T. Lin, "Robust stability of uncertain time-delay systems," Techniques of Automation \& Applications, vol. 46, no. 3, pp. 963-976, 2002.

[25] C. Lin, Q. G. Wang, and H. L. Tong, "Stabilization of uncertain fuzzy time-delay systems via variable structure control approach," IEEE Transactions on Fuzzy Systems, vol. 13, no. 6, pp. 787-798, 2005.

[26] C. Yeroglu, C. Onat, and N. Tan, "A new tuning method for $\mathrm{PI}^{\lambda} \mathrm{D}^{\mu}$ controller," in Proceedings in 2009 International Conference on Electrical and Electronics Engineering, pp. 312-316, Bursa, Turkey, August 2009.

[27] N.-S. Pai, S.-C. Chang, and C.-T. Huang, "Tuning PI/PID controllers for integrating processes with deadtime and inverse response by simple calculations," Journal of Process Control, vol. 20, no. 6, pp. 726-733, 2010.

[28] I. Kaya and D. P. Atherton, Simple Analytical Rules for PI-PD Controllers to Tune Integrating and Unstable Plants, University of Strathclyde Publications, Glasgow, Scotland, 2006.

[29] N. Tan, "Computation of stabilizing PI-PD controllers," International Journal of Control, Automation and Systems, vol. 7, no. 2, pp. 175-184, 2009. 
[30] S. Srivastava and V. S. Pandit, "A PI/PID controller for time delay systems with desired closed loop time response and guaranteed gain and phase margins," Journal of Process Control, vol. 37, no. 1, pp. 70-77, 2016.

[31] C. Onat, S. E. Hamamci, and S. Obuz, "A practical PI tuning approach for time delay systems," IFAC Proceedings Volumes, vol. 45 , no. 14, pp. 102-107, 2012.

[32] C. Onat, "A new concept on PI design for time delay systems: weighted geometrical center," International Journal of Innovative Computing, Information \& Control, vol. 9, no. 4, pp. 1539-1556, 2013.

[33] C. Onat, "WGC based robust and gain scheduling PI controller design for condensing boilers," Advances in Mechanical Engineering, vol. 6, Article ID 659051, 2015.

[34] C. Onat, "A new design method for PI-PD control of unstable processes with dead time," ISA Transactions, vol. 84, no. 1, pp. 69-81, 2019.

[35] A. Shariati, H. D. Taghirad, and A. Fatehi, "A neutral system approach to Ho PD/PI controller design of processes with uncertain input delay," Journal of Process Control, vol. 24, no. 3, pp. 144-157, 2014.

[36] M. M. Ozyetkin, "A simple tuning method of fractional order $\mathrm{PI}^{\lambda}-\mathrm{PD}^{\mu}$ controllers for time delay systems," ISA Transactions, vol. 74, pp. 77-87, 2018.

[37] X. Yu, F. Yang, L. Ou, Q. Wu, and W. Zhang, "General stabilization method of fractional-order $\mathrm{PI}^{\lambda} \mathrm{D}^{\mu}$ controllers for fractional-order systems with time delay," International Journal of Robust and Nonlinear Control, vol. 28, no. 16, pp. 4999-5018, 2018.

[38] I. Kaya, "Simple and optimal PI/PID tuning formulae for unstable time delay processes," in Proceedings of the 2017 10th International Conference on Electrical and Electronics Engineering, pp. 847-851, IEEE, Bursa, Turkey, December 2017.

[39] A. Visioli, "Optimal tuning of PID controllers for integral and unstable processes," IEE Proceedings-Control Theory and Applications, vol. 148, no. 2, pp. 180-184, 2001. 


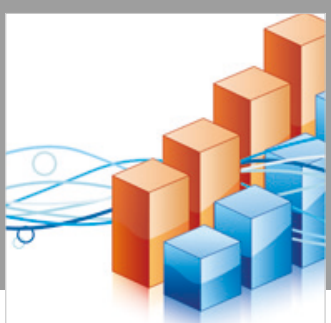

Advances in

Operations Research

\section{-n-m}
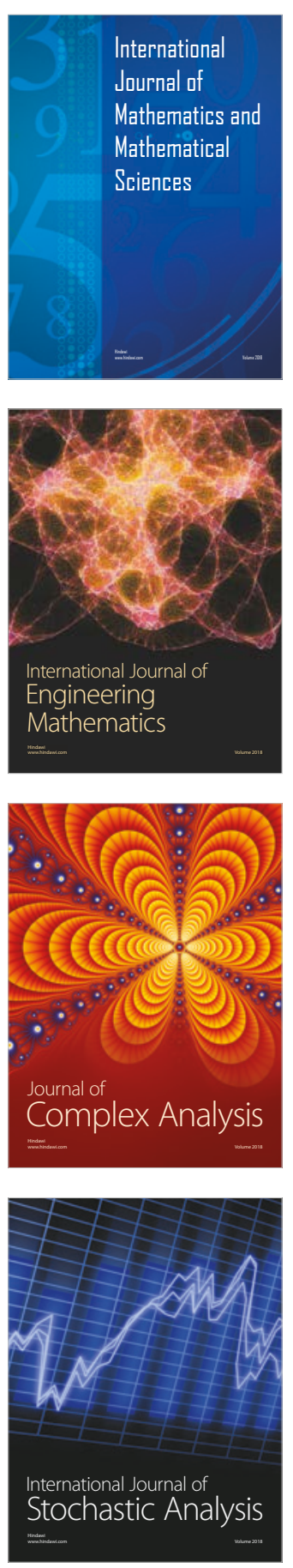
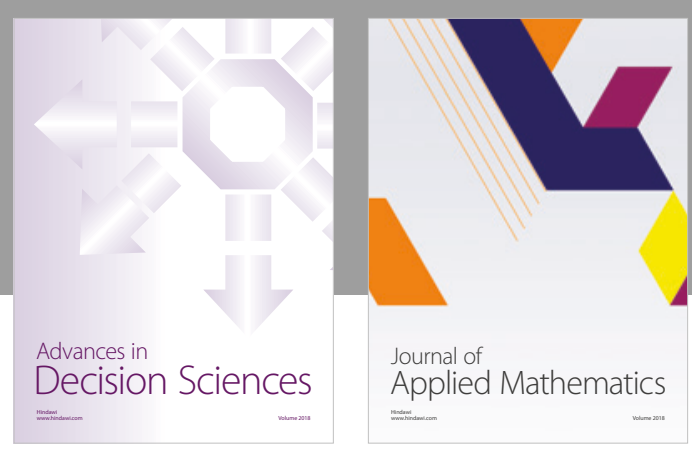

Journal of

Applied Mathematics
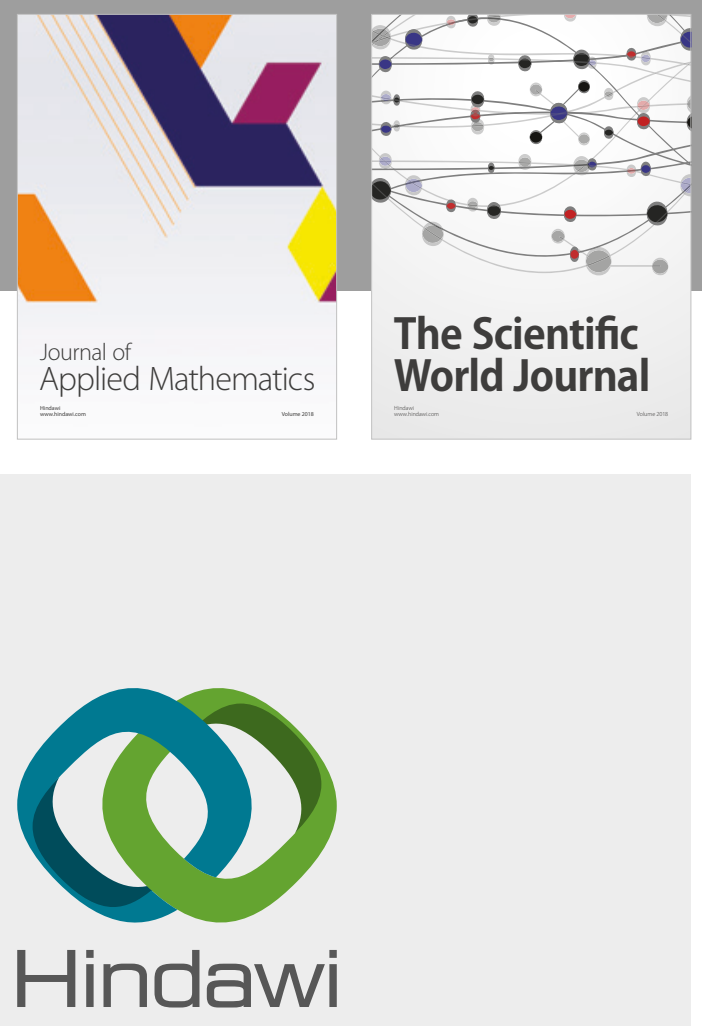

Submit your manuscripts at

www.hindawi.com

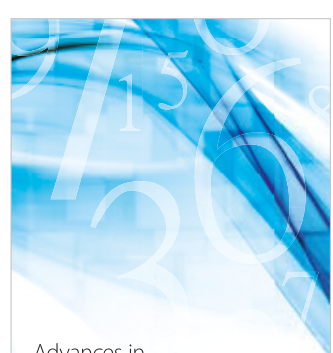

Advances in
Numerical Analysis
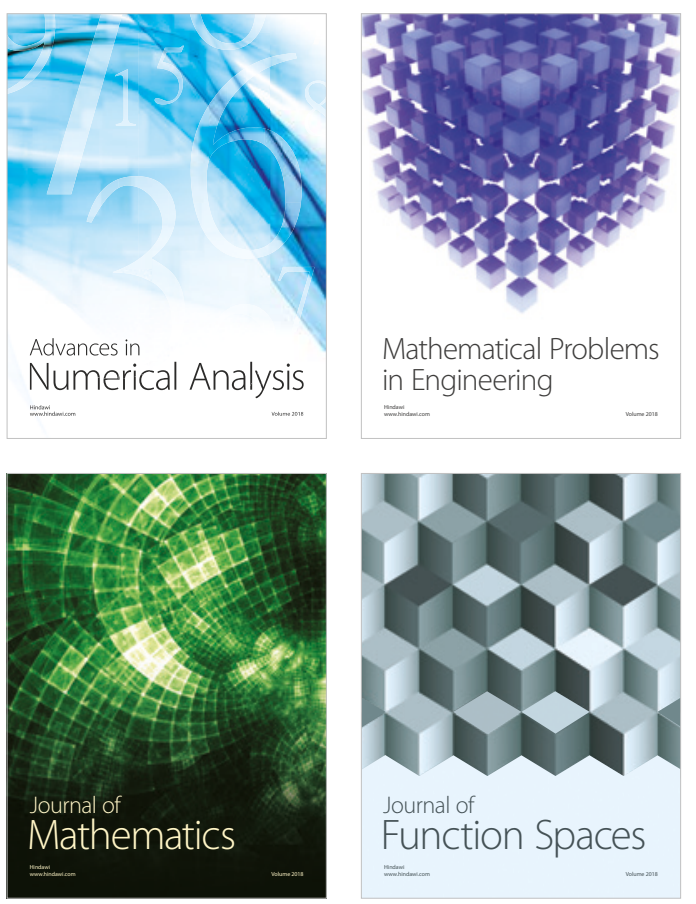

Mathematical Problems in Engineering

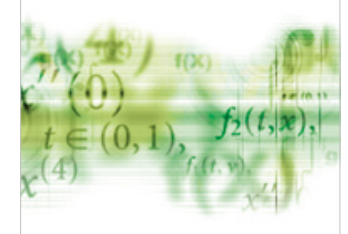

International Journal of

Differential Equations

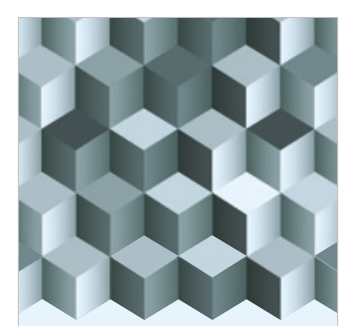

Journal of

Function Spaces
The Scientific

World Journal

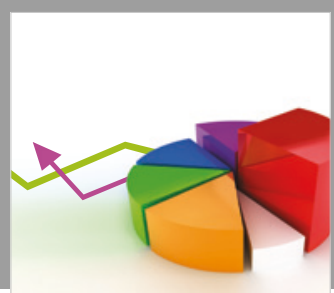

Journal of

Probability and Statistics
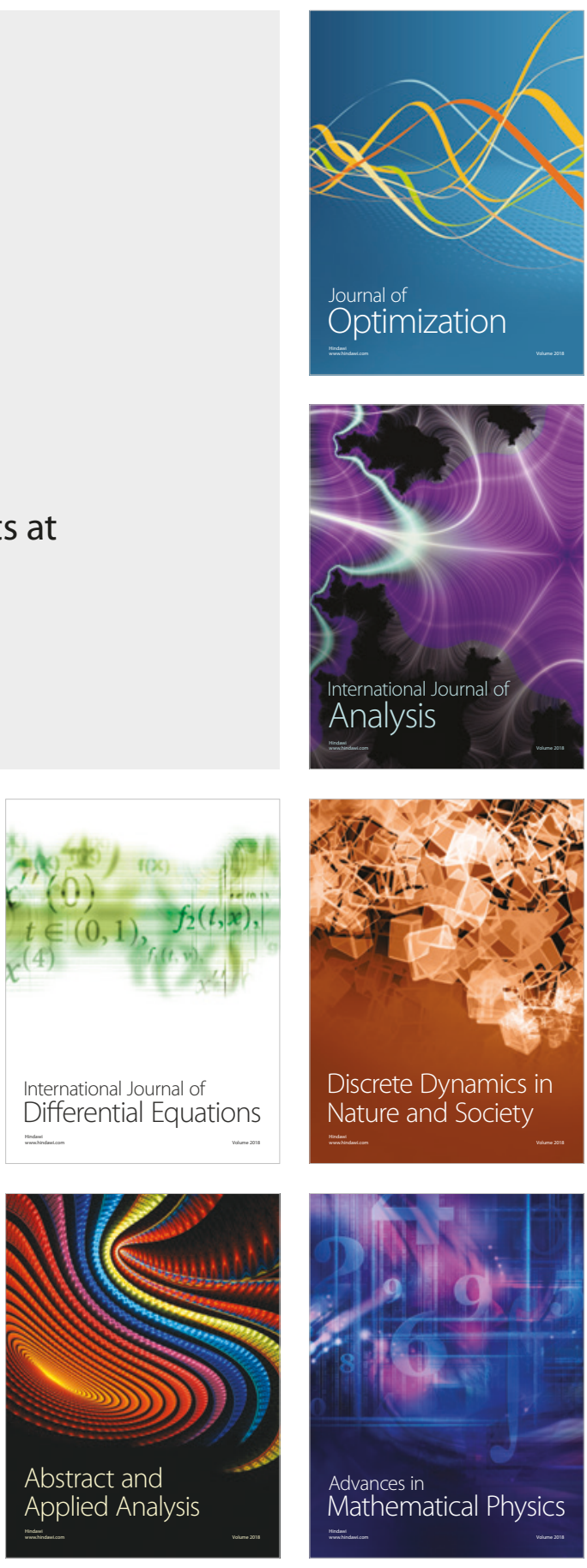